\title{
Probing the stellar wind environment of Vela X-1 with MAXI
}

\author{
C. Malacaria ${ }^{1,2}$, T. Mihara ${ }^{2}$, A. Santangelo ${ }^{1}$, K. Makishima ${ }^{2,3}$, M. Matsuoka ${ }^{2}$, M. Morii $^{2}$, and M. Sugizaki ${ }^{2}$ \\ ${ }^{1}$ Institut für Astronomie und Astrophysik, Sand 1, 72076 Tübingen, Germany \\ e-mail: malacaria@astro.uni-tuebingen.de \\ 2 MAXI team, RIKEN, 2-1 Hirosawa, Wako, 351-0198 Saitama, Japan \\ 3 Department of Physics, The University of Tokyo 7-3-1 Hongo, Bunkyo-ku, 113-0033 Tokyo, Japan
}

Received 20 July 2015 / Accepted 27 January 2016

\begin{abstract}
Context. Vela X-1 is one of the best-studied and most luminous accreting X-ray pulsars. The supergiant optical companion produces a strong radiatively driven stellar wind that is accreted onto the neutron star, producing highly variable X-ray emission. A complex phenomenology that is due to both gravitational and radiative effects needs to be taken into account to reproduce orbital spectral variations.

Aims. We have investigated the spectral and light curve properties of the X-ray emission from Vela X-1 along the binary orbit. These studies allow constraining the stellar wind properties and its perturbations that are induced by the pulsating neutron star.

Methods. We took advantage of the All Sky Monitor MAXI/GSC data to analyze Vela X-1 spectra and light curves. By studying the orbital profiles in the 4-10 and 10-20 keV energy bands, we extracted a sample of orbital light curves ( $15 \%$ of the total) showing a dip around the inferior conjunction, that is, a double-peaked shape. We analyzed orbital phase-averaged and phase-resolved spectra of both the double-peaked and the standard sample.

Results. The dip in the double-peaked sample needs $N_{\mathrm{H}} \sim 2 \times 10^{24} \mathrm{~cm}^{-2}$ to be explained by absorption alone, which is not observed in our analysis. We show that Thomson scattering from an extended and ionized accretion wake can contribute to the observed dip. Fit by a cutoff power-law model, the two analyzed samples show orbital modulation of the photon index that hardens by $\sim 0.3$ around the inferior conjunction, compared to earlier and later phases. This indicates a possible inadequacy of this model. In contrast, including a partial covering component at certain orbital phase bins allows a constant photon index along the orbital phases, indicating a highly inhomogeneous environment whose column density has a local peak around the inferior conjunction. We discuss our results in the framework of possible scenarios.
\end{abstract}

Key words. X-rays: binaries - stars: neutron - stars: winds, outflows - accretion, accretion disks - pulsars: individual: Vela X-1

\section{Introduction}

Vela X-1 is the prototype of the class of wind-fed accreting $\mathrm{X}$-ray binaries. The source has been discovered in 1967 (Chodil et al. 1967) and is located at a distance of $2 \mathrm{kpc}$ (Nagase 1989). It is an eclipsing supergiant high mass X-ray binary (HMXB), consisting of a neutron star (NS) with mass $M_{\mathrm{NS}} \sim 1.77 M_{\odot}$ (Rawls et al. 2011) and a B0.5Ib optical companion, HD 77581, with mass $M_{\mathrm{B}} \sim 23 M_{\odot}$ and radius $R_{\mathrm{B}} \sim 30 R_{\odot}$ (van Kerkwijk et al. 1995).

A sketch of the binary system is shown in Fig. 1. The binary orbit is almost circular (eccentricity $e \sim 0.089$ ), with a period of $\sim 8.9$ d (Kreykenbohm et al. 2008). The NS orbits at a close distance from the companion's surface, $0.7 R_{\mathrm{B}}$ at the apastron, and it is continuously embedded in its strong stellar wind, characterized by $\dot{M}_{\text {wind }}=1.5-2 \times 10^{-6} M_{\odot} \mathrm{yr}^{-1}$ (Watanabe et al. 2006) and terminal velocity $v_{\infty} \sim 1100 \mathrm{~km} \mathrm{~s}^{-1}$ (Prinja et al. 1990). Passing within the NS accretion radius, $r_{\mathrm{acc}}=2 G M_{\mathrm{NS}} / v_{\mathrm{rel}}^{2}$, this wind is accreted onto the compact object, powering the X-ray emission, with luminosity

$L_{\mathrm{X}}=\frac{\left(G M_{\mathrm{NS}}\right)^{3} \dot{M}_{\mathrm{wind}}}{R_{\mathrm{NS}} v_{\mathrm{rel}}^{4} D^{2}}$,

where $G$ is the gravitational constant, $R_{\mathrm{NS}} \sim 10 \mathrm{~km}$ is the NS radius, $v_{\text {rel }}$ the relative velocity of the wind seen from the accretion center, and $D$ the distance of the compact object from the center of the companion.
The observed average X-ray luminosity of $\sim 4 \times 10^{36} \mathrm{erg} / \mathrm{s}$ is consistent with a wind-fed accreting pulsar scenario (see Eq. (1)). However, it is typical of Vela X-1 to show "off-states" in which it becomes faint for several $10^{2}-10^{3} \mathrm{~s}$, and "giant flares", when the source flux suddenly increases up to a factor of 20 (Kreykenbohm et al. 2008; Sidoli et al. 2015). Density inhomogeneities are observed in the matter during the accretion stage (see Martínez-Núñez et al. 2014, and references therein) and are considered a possible reason for the observed X-ray flux variability.

Orbital phase-resolved studies of Vela X-1, both observational and theoretical (see Blondin et al. 1991, and references therein), provide evidence of a strong systematic modulation of the absorbing column density with orbital phase. This is also observed in other HMXBs (see the seminal case of Cen X-3, Jackson 1975, and more recently, the study of IGR J17252-3616, Manousakis et al. 2012). Such an effect is generally attributed to the NS influence on the stellar wind by various mechanisms. These include the formation of a tidal stream trailing the NS (due to the almost filled Roche lobe of the companion), the X-ray photoionization of the wind (which leads to the formation of a Strömgren sphere and a photoionization wake), and an accretion wake that surrounds the compact object (due to the focusing of the stellar wind medium by the NS gravitational influence). A sketch of the binary system where all these structures are illustrated is shown in Fig. 2. 


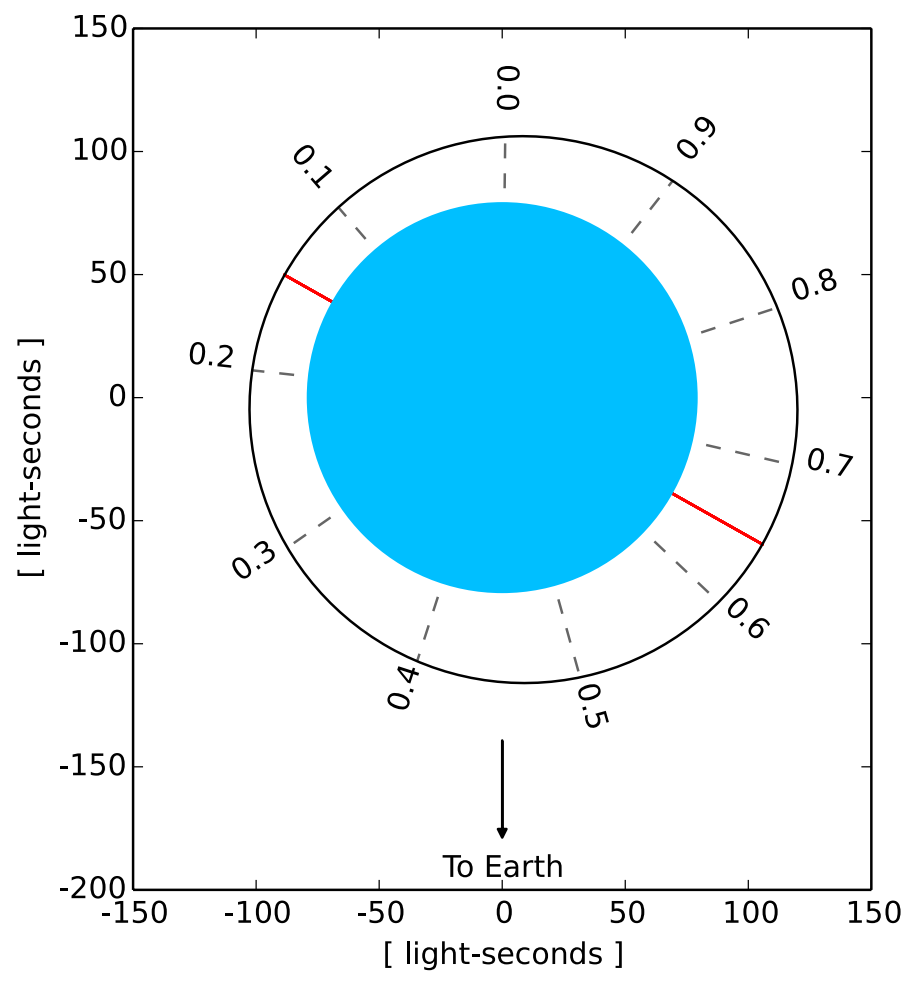

Fig. 1. Orbital sketch of the binary system Vela X-1 as observed from Earth. The blue disk centered at $(0,0)$ is the optical companion HD 77581. The black solid line is the NS elliptical orbit. The gray dashed rays and the respective numbers indicate the orbital phases. Phase zero is taken at the mid-eclipse time. The red straight solid line crossing the ellipse represents the major axis, pointing the periastron and the apastron at the intersections with the NS orbit.

Many authors have reported on the X-ray light curve and spectral modulation of Vela X-1 around the inferior conjunction (e.g., Eadie et al. 1975; Watson \& Griffiths 1977; Nagase 1989). At the same time, the emission from the optical companion also shows modulation with the orbital phase. An absorption feature was reported by Smith (2001), who observed a dip in the Vela X-1 UV light curve around the transit phase $(0.46<\phi<0.70$ in their work, where $\phi=0$ is the mideclipse). Moreover, Goldstein et al. (2004), analyzing spectroscopic Chandra data taken at three different phases (i.e., eclipse $\phi=0, \phi=0.25$ and $\phi=0.5)$, found an absorbed "eclipse-like" spectrum at $\phi=0.5$ in the soft X-ray energy range. Similarly, Watanabe et al. (2006) found an extended structure of dense material that partially covers the X-ray pulsar, which is between the X-ray emitter and the observer at the inferior conjunction. More recently, Naik et al. (2009) analyzed orbital phase-resolved spectra of Vela X-1 observed with RXTE, finding large variation of the absorbing column density along the orbital phase (up to $10^{24} \mathrm{~cm}^{-2}$ ), but their observations do not cover the whole orbit, that is, their data do not include observations between the inferior conjunction and the apastron. Finally, Doroshenko et al. (2013) have shown that the observed increasing absorption at late orbital phase does not agree with expectations for a spherically symmetric smooth wind.

In this paper we present the spectroscopical and light curve analysis of Vela X-1 observed with the Monitor of All-sky X-ray Image (MAXI). Our data show an excellent coverage of the entire orbit and extend over more than four years. We extract a sample of double-peaked orbital profiles in two energy bands, whose dip is around the inferior conjunction. We perform orbital
Table 1. Observation time and ephemeris used for light curves folding.

\begin{tabular}{c|c}
\hline \hline Parameter & Value \\
\hline$\Delta T_{\text {Obs }}[\mathrm{MJD}]^{a}$ & $55133 \div 56674$ \\
$\mathrm{P}[\mathrm{d}]$ & $8.964357 \pm 0.000029$ \\
$T_{\text {Mid-Eclipse }}[\mathrm{MJD}]^{b}$ & 55134.63704 \\
\hline
\end{tabular}

Notes. ${ }^{(a)}$ Time span of the MAXI observations used in this work; (b) Taken as the phase-zero.

phase-averaged and phase-resolved spectroscopy with different models and analyze our results using the most recent stellar wind accretion scenarios.

\section{Observations and data}

The all-sky monitor MAXI (Matsuoka et al. 2009) is operating from the International Space Station (ISS) since August 2009. It consists of two slit cameras working in two complementary energy bands: the Solid-state Slit Camera (SSC, Tomida et al. 2011) in the $0.7-10 \mathrm{keV}$, and the Gas Slit Camera (GSC, Mihara et al. 2011) in the 2-20 keV energy bands. Here we only used archival GSC data. The GSC module is composed of 12 proportional counters, each of which consists of a one-dimensional slit-slat collimator and a proportional counter filled with Xe-gas. Assembled, they allow covering a field of view of $1.5 \times 160 \mathrm{deg}^{2}$, observing about $75 \%$ of the whole sky at each ISS revolution ( 92 $\mathrm{min}$ ), and $95 \%$ per day. The GSC typically scans a point source on the sky for 40-150 s during each ISS revolution (the actual time depends on the source incident angle for each GSC counter). The daily flux source sensitivity at $5 \sigma$ c.l. in the full energy band is about $20 \mathrm{mCrab}$, and the $1 \sigma$ c.l. energy resolution is $18 \%$ at $5.9 \mathrm{keV}$ (Sugizaki et al. 2011). These properties allowed frequently observing Vela X-1 along its orbit and to collecting data from this source for more than four years.

We extracted spectra and light curves of Vela X-1 from GSC data covering almost the whole MAXI operation time, that is, from 55133 to $56674 \mathrm{MJD}$ (see Table 1). Our data were properly corrected to account for the time-dependent effective area of the cameras. All extracted spectra were rebinned to contain at least 200 photons per energy bin. Following the MAXI team recommendation, we added a systematic error at a level of $1 \%$ to the final count rates of the extracted spectra.

\section{Orbital profiles samples analysis}

The mission-long light curve of Vela X-1 as observed by MAXI/GSC is provided by the MAXI team ${ }^{1}$ at different energy ranges. It exhibits a rich variety of timing phenomena. However, we here focus on the orbital properties of the source. We therefore extracted MAXI/GSC light curves with a time resolution of $6 \mathrm{~h}$ in two energy bands, $4-10$ and $10-20 \mathrm{keV}$. Then, we folded the light curves to produce average orbital profiles in the two chosen energy bands, using the orbital ephemeris from Kreykenbohm et al. (2008) that we report in Table 1. The phase zero is taken at the mid-eclipse time (see Fig. 1). The resulting orbital light curves are shown in Fig. 3, where the strongest

The entire MAXI/GSC VelaX-1 light curve can be
explored at http://maxi.riken.jp/top/index.php?
cid=1\&jname=J0902-405




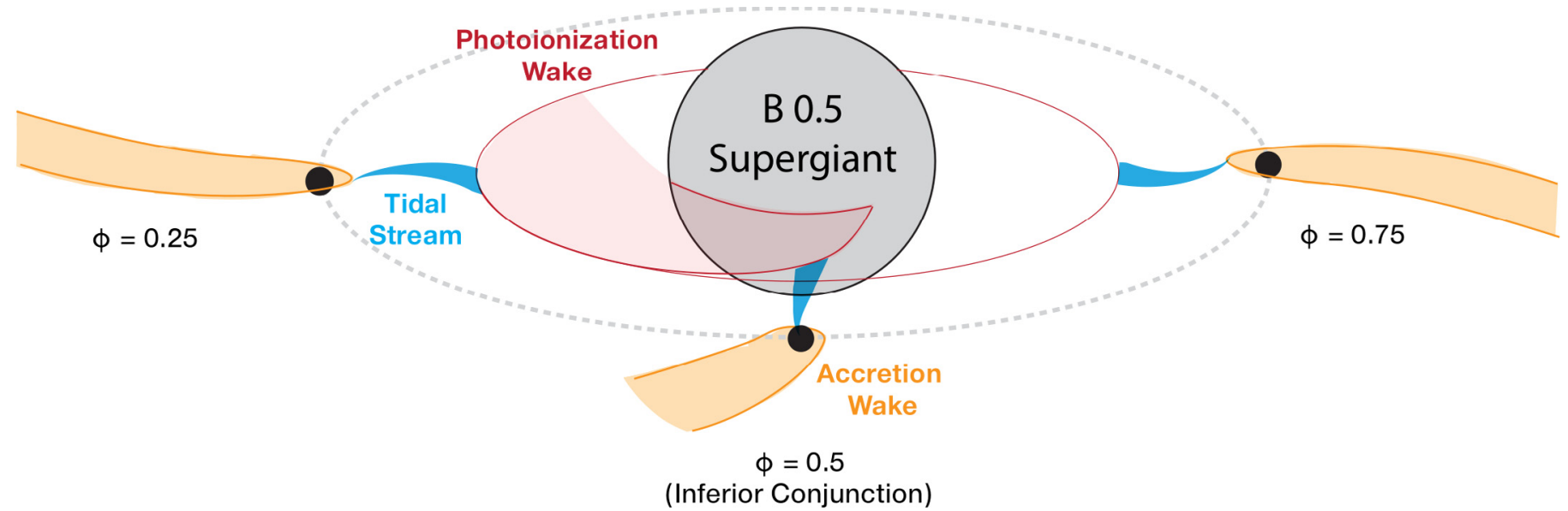

Fig. 2. Sketch of the binary system Vela X-1 where both radiative and gravitational effects are illustrated at three different orbital phases. The photoionization wake is represented as a disk-like structure to show both the cumulative effect along one entire orbit (the red ring) and the more localized effect (the fainter red tail) trailing the NS at the inferior conjunction. A clumpy stellar wind, not shown in the picture, may also be taken into account for a more complete scenario.

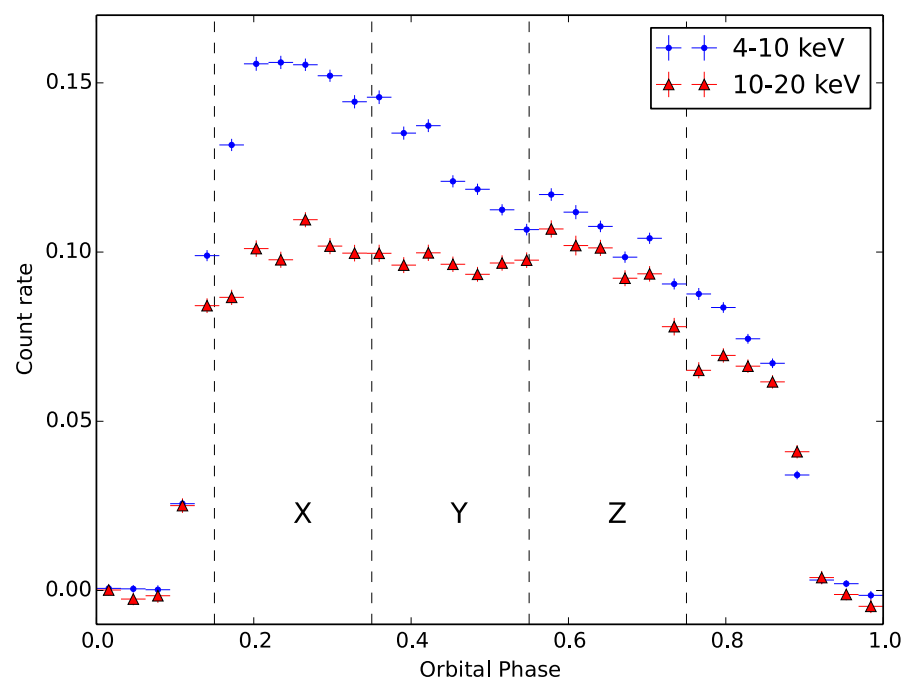

Fig. 3. Vela $X-1$ folded orbital light curves in $4-10$ (blue dots), and $10-20 \mathrm{keV}$ (red triangles) energy ranges. The horizontal bars indicate the phase-bin. The mid-eclipse time is taken as the phase zero. The three orbital phase bins $(X, Y$, and $Z)$ used for the phase-resolved spectroscopy of the double-peaked sample are indicated (see text).

changes are observed in the softer band light curve. This is explained as strong absorption column modulation along the binary orbit.

Successively, we considered the difference between individual orbital light curves (defined as the members of the sample) and the average orbital profile (defined as the template). To obtain a smooth template of the two folded light curves, we interpolated with a cubic spline function between all the pairs of consecutive data points (the knots) of the light curves.

From our line of sight, the accretion wake effects are more prominent for about 0.2 in orbital phase (i.e., about 1.5 days) around the inferior conjunction (see Fig. 1). To properly sample these effects, we defined three orbital phase bins that we call $X, Y$, and $Z$, corresponding to phase intervals $0.15-0.35$, $0.35-0.55$, and $0.55-0.75$, respectively (see Fig. 3). For analogous reasons we imposed for each member a minimum number of data points in each phase bin equal to 4 (i.e., $1 \mathrm{~d}$ observation), which leaves us with a total of 84 statistically acceptable cycles.
This allowed us to perform a systematic analysis of those members that differ from the template in each of the three defined phase bins. For the systematic comparison of the members' profile against the template, we considered only the relative shape, avoiding any bias from flux variation. For this reason, we normalized each member with a normalization factor equal to the ratio of the average count rate between $0.10-0.85$ and the template average count rate in the same phase interval. Successively, we subtracted the count rates of the template from the count rates of each member in the $X, Y$, and $Z$ phase bins. To evaluate the template and the member count rate in each phase bin, we calculated the median of the data points. The obtained count rate difference is referred to as the residuals with respect to the template. The residuals of the members from the template for the $Y$ phase-bin in the 4-10 keV energy band are shown in Fig. 4. This plot shows a scattered distribution of the residuals, as expected because of the high variability of the source. However, the plot also clearly shows that residuals tend to cluster toward negative values. Therefore, we performed a new analysis and stacked only those members that had high $(>30 \%)$ negative residuals (i.e., $<-0.0345 \mathrm{cnt} / \mathrm{s}$ ) in the $Y$ phase bins and at the same time low $(<30 \%)$ residuals in the $X$ and $Z$ phase bins. This allowed us to obtain a new average orbital profile in the $4-10 \mathrm{keV}$ energy band, made of 12 out of 84 total orbital cycles (i.e., about $15 \%$ of the analyzed cycles). This is shown in Fig. 5. Using a different definition of the residuals, where the ratio between the median values is taken (instead of their difference), shows the very same trend as that in Fig. 4, and leads to the same selected profiles.

Since our normalization procedure could remove fluxdependent features, we carefully verified that these selected profiles were not associated with a specific luminosity level before normalization. In addition, for all of these 12 selected profiles, the null hypothesis was tested against the template with normality tests $\left(\chi^{2}\right.$, Kolmogorov-Smirnov, Shapiro-Wilk) and was rejected at a significance level higher than $3 \sigma$. Furthermore, they all show a median value in the $Y$ phase-bin that deviates at least $3 \sigma$ from the template. These profiles are characterized by a double-peak shape and a flux drop around the inferior conjunction. We refer to them as the double-peaked sample.

Similar results are obtained if the same procedure is performed in the $10-20 \mathrm{keV}$ energy band. In this case, the selected 


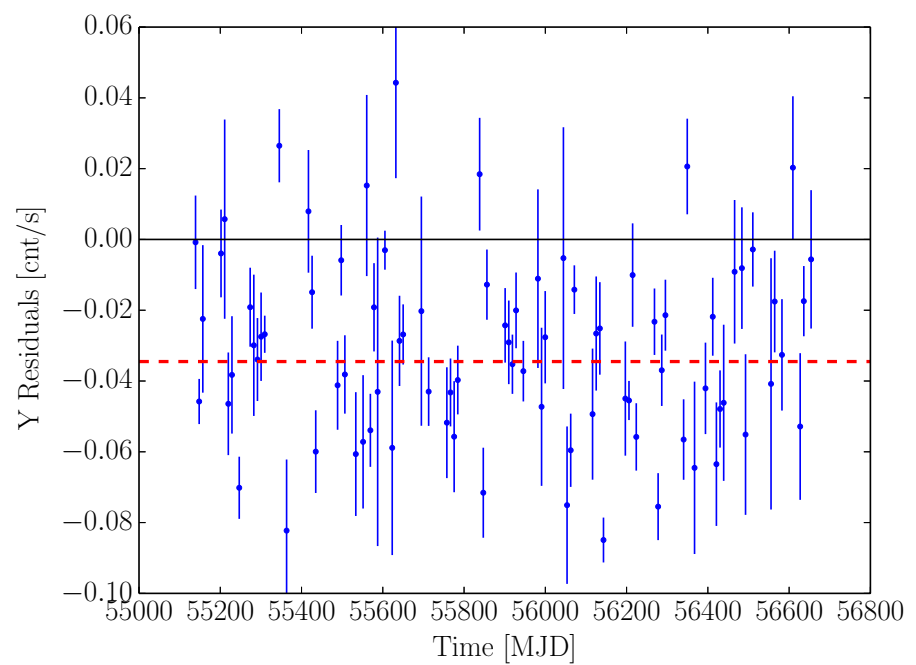

Fig. 4. Residuals of single orbital profiles from the template in the 4-10 keV energy band for the $Y$ phase-bin (see text). The residuals are compared to the observation time on the $x$-axis. The thin black line is the zero-value, while the red dashed line is the $30 \%$ limit below which the selection criterion in the $Y$ phase-bin is fullfilled. Error bars correspond to the sample standard deviation $(\sigma / \sqrt{N}$, with $N$ number of data points). Not all the profiles below the red dashed line are selected as double-peaked profiles because of the conditions imposed on the $X$ and $Z$ phase-bins.

profiles are 11 out of 84 . The overall profile shape is very similar to the profile obtained in the $4-10 \mathrm{keV}$ energy band, with a double-peak morphology and a dip around the inferior conjunction. The extracted sample in the $10-20 \mathrm{keV}$ energy band is shown in Fig. 5.

Finally, we extracted another independent sample in the two energy bands by stacking all the statistically acceptable orbital profiles except for the double-peaked members in each respective energy band. We refer to this sample as the standard sample. The standard sample orbital profiles in the two energy bands are shown with dashed lines in Fig. 5.

\section{Phase-averaged spectral analysis}

The orbital phase-averaged spectral analysis of Vela X-1 was performed separately for the two samples defined in Sect. 3. Because the physical problem is complex, a fully physical model of the X-ray production mechanism in accreting X-ray pulsars has not been implemented so far. Therefore, an empirical model has to be used. Following the common empirical model used in the literature (see, e.g., Schanne et al. 2007), we adopted a cutoff power-law model (cutoffpl in XSPEC) to which a blackbody component was added to account for soft excess (see Hickox et al. 2004 and references therein). On the other hand, to account for the expected inhomogeneous structure of the ambient wind in Vela X-1, we also tested the partial covering model (pcfabs*powerlaw in XSPEC) characterized by a local absorbing component $\left(N_{\mathrm{H}}^{\mathrm{pc}}\right)$ that affects only a fraction $f$ of the original spectrum. This model has also been successfully used to fit NuStar data of Vela X-1 (Fürst et al. 2014). To both models, photoelectric absorption by neutral matter (Morrison \& McCammon 1983) was added, to account for the absorption column density $\left(N_{\mathrm{H}}\right)$ along the entire line of sight. We assumed solar abundances by Anders \& Grevesse (1989) for this. Using the metal abundances of Kaper et al. (1993) for HD 77581 (i.e., sub-solar $\mathrm{C}$, and super-solar $\mathrm{N}, \mathrm{Al}$ ) does not affect our results, which

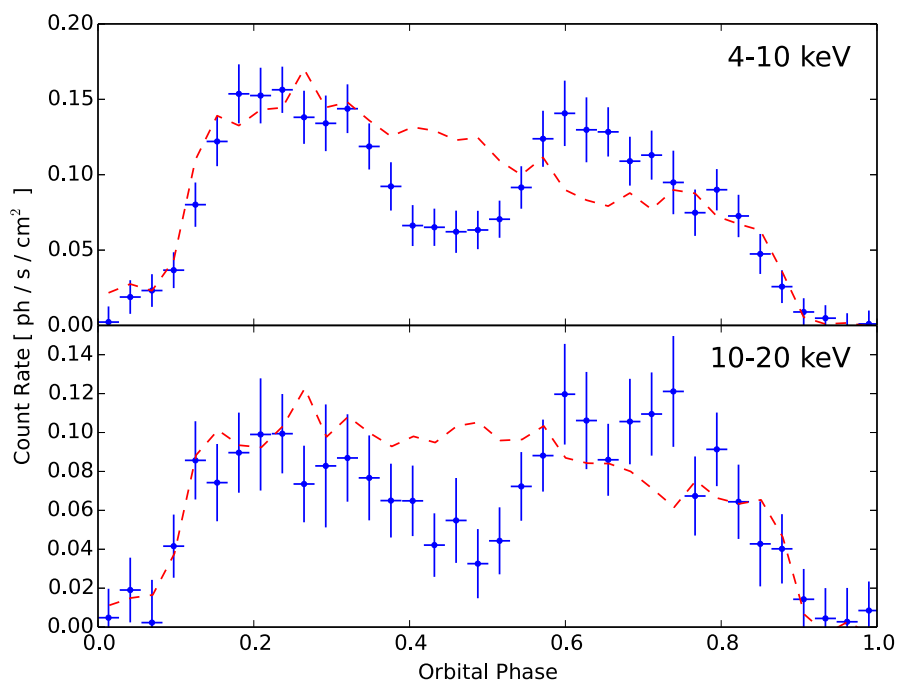

Fig. 5. Average orbital profiles of the double-peaked and of the standard samples in 4-10 keV (top) and 10-20 keV (bottom). The blue dots are the data points obtained by stacking the memebers with large residuals in the $Y$ phase bin (see text). The vertical error bars correspond to the standard deviation of the average of the count rates in each phase bin, while the horizontal bars indicate the phase-bin width. The red dashed lines represents the function interpolated between the data points of the standard samples in each energy band.

are well consistent within $1 \sigma$. Therefore, we used solar abundances throughout the rest of the analysis. We also added two iron Gaussian line components (Odaka et al. 2013), $\mathrm{K} \alpha$ and $\mathrm{K} \beta$ at 6.4 and $7.08 \mathrm{keV}$. The same components were included in the models used for the phase-resolved spectroscopy. The results of the fits are given in Table 2. Both models return acceptable fits for the standard and the double-peaked sample. However, for the standard sample, the partial covering model describes the data not as well as the cutoff power-law model.

\section{Phase-resolved spectral analysis}

\subsection{Orbital phase-resolved spectroscopy of the double-peaked sample}

To investigate the nature of the dip in the double-peaked sample, we extracted X-ray spectra of Vela X-1 in the $X, Y$ and $Z$ orbital phase bins. For this, we filtered MAXI/GSC data with GTIs corresponding to the three phase bins in the double-peaked profiles (see Sect. 3). Based on the results from Sect. 4, we used the same models as were applied in the phase-averaged case to fit the orbital phase-resolved spectra: (a) a cutoff power-law model plus a blackbody component, and (b) a partial covering model. To avoid unphysical best-fit solutions when model (a) was used, we fixed the cutoff energy and the blackbody temperature to their respective phase-averaged values. For model (a) we also compared the results for a fixed (to the phase-averaged value, $\Gamma=0.27$, see Table 2) or free photon index. We found that the model with free photon index is statistically preferable $\left(\Delta \chi^{2}>20\right)$. Furthermore, fitting the $Y$ phase bin spectrum, the model with a fixed photon index leads to unphysically high values of the blackbody normalization (to compensate for the soft excess). Thus, we did not consider the cutoff power-law model with a fixed photon index a viable model in our analysis (similar considerations hold for the phase-resolved spectroscopy of the standard sample, see Sect. 5.2). It is often possible to find a statistically equivalent solution for model (b) where the partial covering component is 


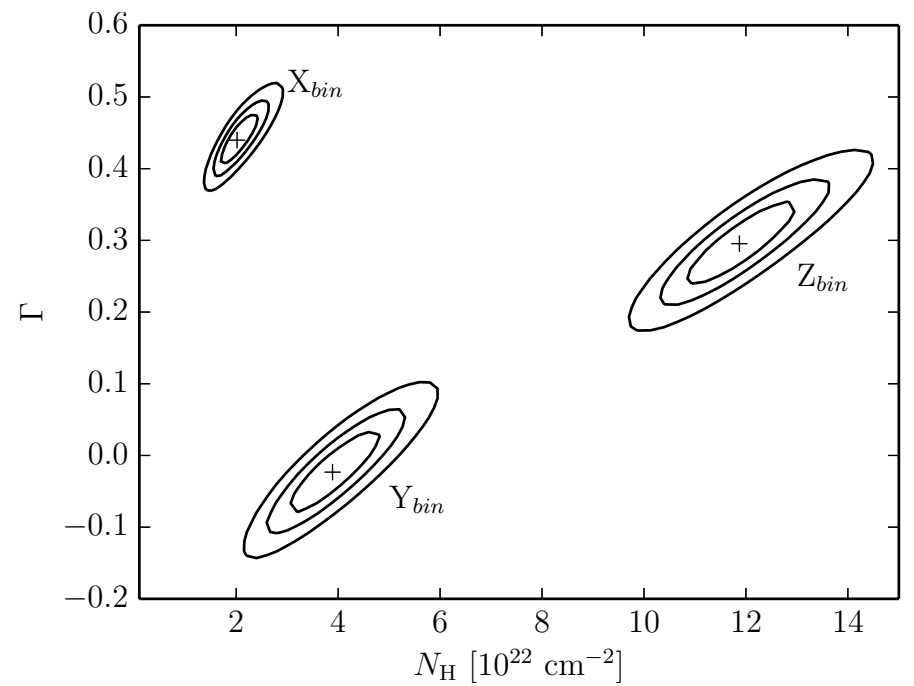

Fig. 6. $\chi^{2}$ contour plots for the Vela X-1 double-peaked sample fitted with an absorbed cutoff power-law model including a blackbody component (model $a$, see Table 3 ). The ellipses are $\chi^{2}$-contours for two parameters $\left(N_{\mathrm{H}}\right.$ and $\Gamma$ ). The contours correspond to $\chi_{\min }^{2}+1.0$ (the projections of this contour to the parameter axes correspond to the $68 \%$ uncertainty for one parameter of interest), $\chi_{\min }^{2}+2.3$ (68\% uncertainty for two parameters of interest), and $\chi_{\min }^{2}+4.61$ (90\% uncertainty for two parameters of interest). The respective orbital phase bins are indicated.

not necessary. We tested the significance of the partial covering component with the F-test, including such a component only when it was significant at a $99 \%$ c.l. Otherwise, we preferred the solutions where the partial covering component was not included. The same criterion was adopted for the standard sample case (see Sect. 5.2)

Models (a) and (b) both return a good fit, and the results are shown in Table 3. Model (a) shows orbital modulation of the spectral photon index $\Gamma$. To better explore the spectral variation, we produced $\chi^{2}$-contour plots of $\Gamma$ and $N_{\mathrm{H}}$ in the three phase bins (see Fig. 6).

Conversely, model (b) does not show photon index modulation along the orbital phase, although it shows variation of the column density. A plot of the double-peaked phase-resolved spectra fit with the partial covering model is shown in Fig. 8.

\subsection{Orbital phase-resolved spectroscopy of the standard sample}

Similarly to the double-peaked sample, we performed orbital phase-resolved spectroscopy of the standard sample. However, for this sample, the statistics is much higher than in the previous case, so that a finer binning is possible. We divided the standard sample profile into seven phase bins, each one wide 0.1 in phase, from 0.1 to 0.8 . Phase bins around the eclipse time are not suitable for our purpouses and suffer from lower statistics, therefore they were not analyzed in our work. The analyzed phase bins are labeled $\phi_{\mathrm{i}}$, with $i=1, \ldots, 7$. Then we filtered our data with GTIs corresponding to the seven orbital phase bins and extracted orbital phase-resolved spectra of the standard sample. Based on the results from Sect. 4, we fit the phase-resolved spectra with the same two models: (a) a cutoff power-law with a blackbody component, and (b) a partial covering model. To avoid unphysical best-fit solutions, we fixed the cutoff energy and the blackbody temperature of model (a) to their respective phase-averaged value. As already mentioned in Sect. 5.1, model (b) returns two

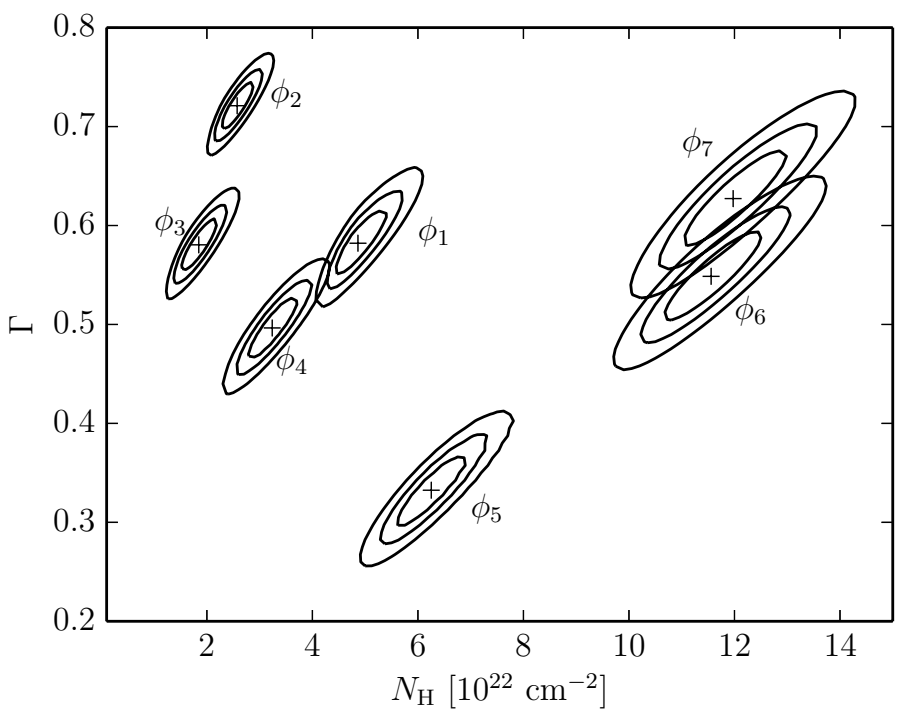

Fig. 7. $\chi^{2}$ contour plots for the Vela X-1 standard sample fitted with an absorbed cutoff power-law model including a blackbody component (see Table 4). Symbols are the same as in Fig. 6. The respective orbital phase bins are indicated.

statistically equivalent solutions: one where the partial covering component is statistically significant, and the other where the same component is not necessary. In such cases, we adopted the solution in which the partial covering component was not required, unless it was significant at a $99 \%$ c.l. The results are given in Table 4. All phase-resolved spectra return good fits for each of the two tested models. However, the cutoff power-law model with blackbody shows a marked modulation of the spectral photon index $\Gamma$ along the orbital phase. The trend of this modulation is similar to that observed for the double-peaked sample (see Sect. 5.1). For a deeper investigation of these results, $\chi^{2}$-contour plots of $\Gamma$ and $N_{\mathrm{H}}$ were produced (see Fig. 7).

The partial covering model shows only marginal variation of the photon index $\Gamma$, while the column density clearly changes along the orbital phase, and the covering fraction is consistent with a constant value. A plot of the standard sample phaseresolved spectra fit with the partial covering model is shown in Fig. 9.

\section{Discussion}

The main observational results of our work can be summarized as follows:

- About $15 \%$ of the orbital light curves in Vela X-1 shows a double-peaked orbital profile, with a dip around the inferior conjunction, both in the $4-10 \mathrm{keV}$ and $10-20 \mathrm{keV}$ energy bands;

- orbital phase-resolved spectra of both the double-peaked and the standard sample are well fit by a cutoff power-law model and by a partial covering model;

- the cutoff power-law model leads to orbital modulation of both the spectral photon index and the column density;

- a partial covering model fits the data equally well. No orbital modulation of the spectral photon index is observed in this case. In contrast, orbital modulation of the column density is observed.

In the following we discuss our results within the context of the most recent wind accretion scenarios that have been proposed for Vela X-1. 
Table 2. Best-fit spectral parameters of the orbital phase-averaged spectra from the double-peaked and standard samples.

\begin{tabular}{l|cc|cc}
\hline \hline Model & \multicolumn{2}{|c|}{ Cutoff PL $+\mathrm{BB}$} & \multicolumn{2}{c}{ Part. Cov. } \\
\hline Sample & Double-peaked & Standard & Double-peaked & Standard \\
\hline$N_{\mathrm{H}} / 10^{22} \mathrm{~cm}^{-2}$ & $3.91_{-0.36}^{+0.43}$ & $4.29_{-0.30}^{+0.31}$ & $2.55_{-2.55}^{+2.18}$ & $2.02_{-0.79}^{+0.68}$ \\
$N_{\mathrm{H}}{ }^{p c} / 10^{22} \mathrm{~cm}^{-2}$ & - & - & $14.9_{-5.4}^{+16.0}$ & $12.7_{-1.7}^{+2.5}$ \\
Cov. Fract. ${ }^{p c}$ & - & - & $0.63_{-0.18}^{+0.21}$ & $0.64_{-0.06}^{+0.07}$ \\
$\Gamma$ & $0.27_{-0.03}^{+0.04}$ & $0.48_{-0.06}^{+0.06}$ & $1.09_{-0.07}^{+0.10}$ & $1.13_{-0.03}^{+0.03}$ \\
$E_{\text {cut }}[\mathrm{keV}]$ & $15.3_{-3.4}^{+0.4}$ & $20.1_{-2.0}^{+2.5}$ & - & - \\
$k T_{\mathrm{BB}}[\mathrm{keV}]$ & $0.16_{-0.04}^{+0.04}$ & $0.14_{-0.08}^{+0.06}$ & - & - \\
$\chi^{2} /$ d.o.f. $=\chi_{\text {red }}^{2}$ & $285 / 311=0.92$ & $393 / 351=1.12$ & $318 / 311=1.02$ & $480 / 352=1.36$ \\
\hline
\end{tabular}

Notes. The errors correspond to the $1 \sigma$-uncertainties. ${ }^{(p c)}$ Partial Covering Model.

Table 3. Best-fit spectral parameters of the phase-resolved spectra from the double-peaked sample.

\begin{tabular}{l|cc|cc|cc}
\hline \hline Phase-bins & \multicolumn{2}{|c|}{$X$} & \multicolumn{2}{c|}{$Y$} & \multicolumn{2}{c}{$Z$} \\
\hline Model & Cutoff PL + BB & Part. Cov. & Cutoff PL + BB & Part. Cov. & Cutoff PL + BB & Part. Cov. \\
\hline$N_{\mathrm{H}} / 10^{22} \mathrm{~cm}^{-2}$ & $2.02_{-0.31}^{+0.41}$ & $3.68_{-2.16}^{+0.29}$ & $3.89_{-0.87}^{+0.93}$ & $2.49_{-2.49}^{+2.07}$ & $11.9_{-1.0}^{+1.1}$ & $16_{-3}^{+1}$ \\
$N_{\mathrm{H}}{ }^{p c} / 10^{22} \mathrm{~cm}^{-2}$ & - & - & - & $16.1_{-5.3}^{+11.3}$ & - & - \\
Cov. Fract. $^{p c}$ & - & - & - & $0.64_{-0.09}^{+0.17}$ & - & - \\
$E_{\text {cut }}[\mathrm{keV}]$ & 15.3 (fixed) & - & 15.3 (fixed) & - & 15.3 (fixed) & - \\
$\Gamma$ & $0.44_{-0.03}^{+0.04}$ & $1.12_{-0.04}^{+0.02}$ & $-0.02_{-0.06}^{+0.06}$ & $0.91_{-0.11}^{+0.29}$ & $0.29_{-0.06}^{+0.06}$ & $1.10_{-0.05}^{+0.05}$ \\
Norm $_{\mathrm{PL}}$ & $0.110_{-0.008}^{+0.009}$ & $0.235_{-0.017}^{+0.014}$ & $0.003_{-0.005}^{+0.005}$ & $0.129_{-0.038}^{+0.151}$ & $0.089_{-0.01}^{+0.015}$ & $0.256_{-0.031}^{+0.042}$ \\
$k T_{\mathrm{BB}}[\mathrm{keV}]^{b}$ & 0.16 (fixed) & - & 0.16 (fixed) & - & 0.16 (fixed) & - \\
$\chi^{2} /$ d.o.f. $=\chi_{\text {red }}^{2}$ & $113 / 121=0.93$ & $121 / 120=1.00$ & $113 / 108=1.05$ & $117 / 107=1.09$ & $101 / 102=0.99$ & $113 / 101=1.11$ \\
\hline
\end{tabular}

Notes. The errors correspond to the $1 \sigma$-uncertainties. ${ }^{(p)}$ Partial Covering Model. ${ }^{(b)}$ Blackbody component.

\subsection{Double-peaked orbital light curve}

Indications of a double-peaked orbital light curve in Vela X-1 have already been reported by earliest observations of the source with Ariel V (Eadie et al. 1975; Watson \& Griffiths 1977). Based on Tenma data, Nagase (1989) found an increase of the column density along the NS orbit, in particular after the transit phase. Blondin et al. (1991) took both gravitational and radiative effects into account and reproduced the observed orbital phase dependence of the column density. They found that an accretion wake that extends toward the observer's line of sight at the inferior conjunction produces a local peak in the column density absorption along the binary orbit.

Intriguing evidence on the accretion wake in Vela X-1 was obtained by Smith (2001), who inferred that a hot source around the transit phase of the NS is present by analyzing He $\lambda 1640 \mathrm{ab}-$ sorption line strengths. Based on Chandra X-ray data, Watanabe et al. (2006) also have inferred the presence of a dense cloud obscuring the compact source at the inferior conjunction. All these results are tightly related to the observational features reported in this work.

The sample of double-peaked orbital profiles, obtained from the very long MAXI/GSC monitoring of Vela X-1, shows a remarkable dip at phase $Y$, also in the $10-20 \mathrm{keV}$ band. This is challenging to explain solely by invoking absorption from neutral matter. The X-ray flux observed at the dip phase is about half of the flux in the standard sample at the same phase (see Fig. 3). To halve a luminosity of $\sim 4 \times 10^{36} \mathrm{erg} / \mathrm{s}$ in the $10-20 \mathrm{keV}$ band, a column density value of $N_{\mathrm{H}} \sim 2 \times 10^{24} \mathrm{~cm}^{-2}$ is needed, $\sim 10$ times higher than what is observed in our study.

In addition, hydrodynamical simulations of stellar winds in HMXBs (Blondin et al. 1991; Manousakis et al. 2012) predict a local peak of the column density $N_{\mathrm{H}}$ around the inferior conjunction on the order of $10^{23} \mathrm{~cm}^{-2}$. This agrees with our observations. We also mention that the INTEGRAL analysis of Fürst et al. (2010) highlights a possible dip-like feature around the transit in the phase-resolved flux histograms of Vela X-1 at $20-60 \mathrm{keV}$, that is, at an energy band that is almost unaffected by absorption. Our conclusion is therefore that some additional mechanism must be at work that contributes to the flux reduction at the inferior conjunction, and that is triggered only in a limited sample of orbital cycles.

A contribution to the observed reduced flux could come from the Compton scattering at the low-energy limit, where the cross section is characterized by only a weak dependence on the photon energy and can be approximated by the Thomson scattering cross section $\left(\sigma_{\mathrm{T}}=6.65 \times 10^{-25} \mathrm{~cm}^{2}\right)$. To efficiently scatter X-rays from the accreting pulsar, the surroundings of the compact object need to be ionized and dense enough. Hydrodynamical 2D and 3D simulations of the stellar wind in Vela X-1 by Mauche et al. (2008) showed that the interaction of the wind with the NS leads to strong variations of the wind parameters. These authors found that the accretion wake reaches high temperatures $\left(\sim 10^{8} \mathrm{~K}\right)$ and a high ionization parameter $\left(\xi=L_{x} /\left(n R_{\text {ion }}^{2}\right) \sim 10^{4} \mathrm{erg} \mathrm{cm} \mathrm{s}^{-1}\right.$, where $n$ is the number density at a given point, while $R_{\text {ion }}$ is the distance from this point to 


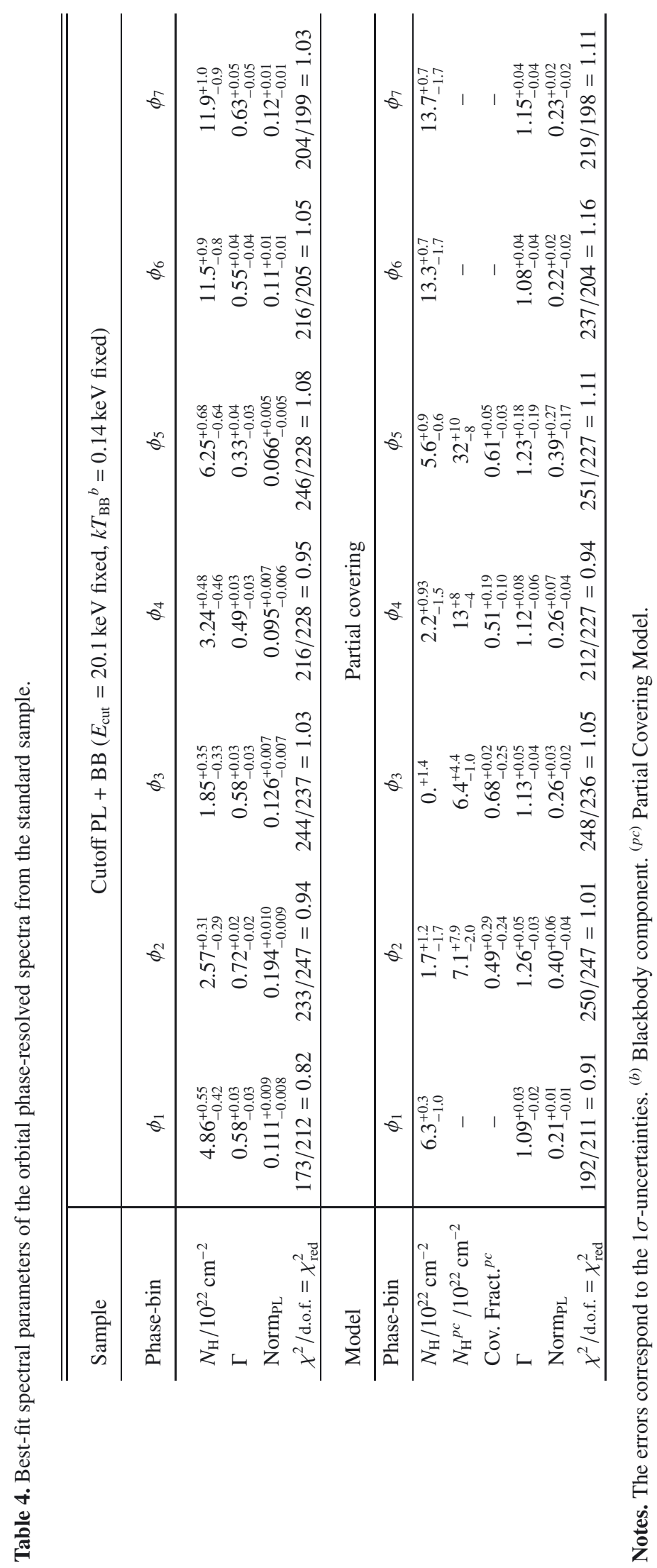




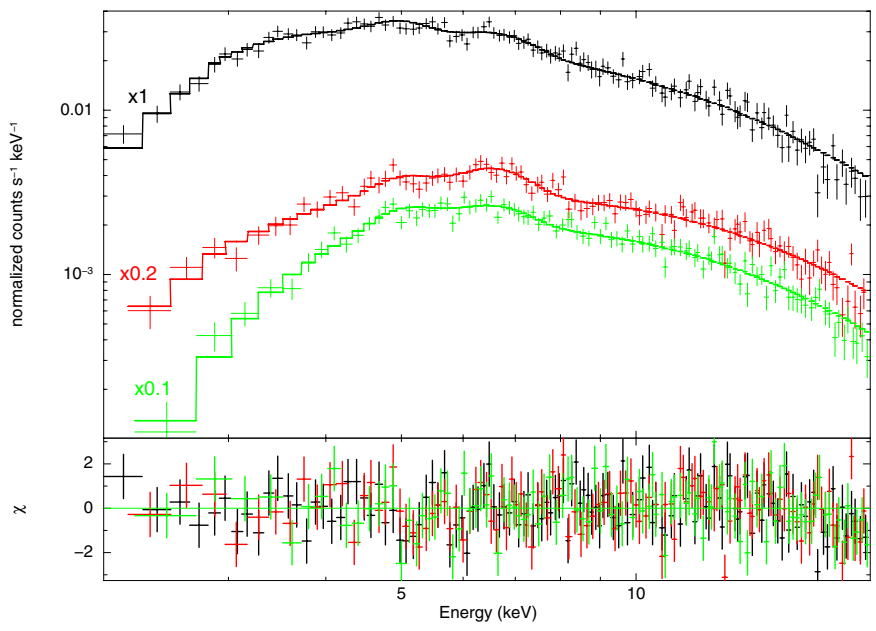

Fig. 8. Orbital phase-resolved spectra of the double-peaked sample fitted with a partial covering model, when significant (see text). Top panel (from top to bottom): data and folded model of the three spectra in phase bins $X, Y$, and $Z$. The spectra have been multiplied by the factor shown above each curve on the left side for better visualization. Bottom panel: residuals for the best-fit model. See Table 3 for all spectral parameters.

the X-ray source). The accretion wake extends to scales on the order of the optical companion size $\left(R \sim 10^{12} \mathrm{~cm}\right)$ with a number density of $\sim 10^{9-12} \mathrm{~cm}^{-3}$, which, taking an average value of $n \sim 10^{10} \mathrm{~cm}^{-3}$, leads to a column density of $\sim 10^{23} \mathrm{~cm}^{-2}$. This is similar to the values obtained from our spectral analysis of the double-peaked sample in the $Y$ phase bin (see Sect. 6.2.2 and Table 3). Moreover, the bremsstrahlung cooling time is about $10^{11} \sqrt{T} / n$ s (Rybicki \& Lightman 1979) that is, $\sim 10^{5} \mathrm{~s}$, or about one day, which agrees with the observed time duration of the dip. In other words, the gas stays ionized for a time long enough to allow Thomson scattering. The ionized gas is not traced by the spectral absorption in the soft X-ray band. Nonetheless, its electron density $n$ is high enough to result in an optical depth value of $\tau=n l \sigma_{\mathrm{T}}=0.5-0.7$ (where $l=2 \times 10^{12} \mathrm{~cm}$ is the length of the accretion wake). Thus, when the ionized stream of the accretion wake passes through the observer's direction, that is, during the transit, a substantial part of the X-ray radiation can be efficiently scattered out of our line of sight. This scenario is further supported by the normalization of the power-law component, which appears to be modulated along the orbit (see Table 3), indicating an intrinsically dimmer flux at the inferior conjunction (although large parameter uncertainties need to be considered)

Stellar-wind variability may then be responsible for the modifications of the accretion wake, which in turn modify the orbital light curve shape, alternating between a double-peaked and a standard morphology. We therefore conclude that the ionized gas in the hot accretion wake, combined with its high column density, is a possible cause for the dip that is observed at the inferior conjunction in the double-peaked sample light curves.

\subsection{Spectral results}

\subsubsection{Orbital phase-averaged spectroscopy}

Orbital phase-averaged spectra in the $2-20 \mathrm{keV}$ energy range of both the double-peaked and the standard sample were modeled with a cutoff power-law model, adding a blackbody component to account for the soft excess, and with partial covering (Table 2). For the double-peaked sample, both models return

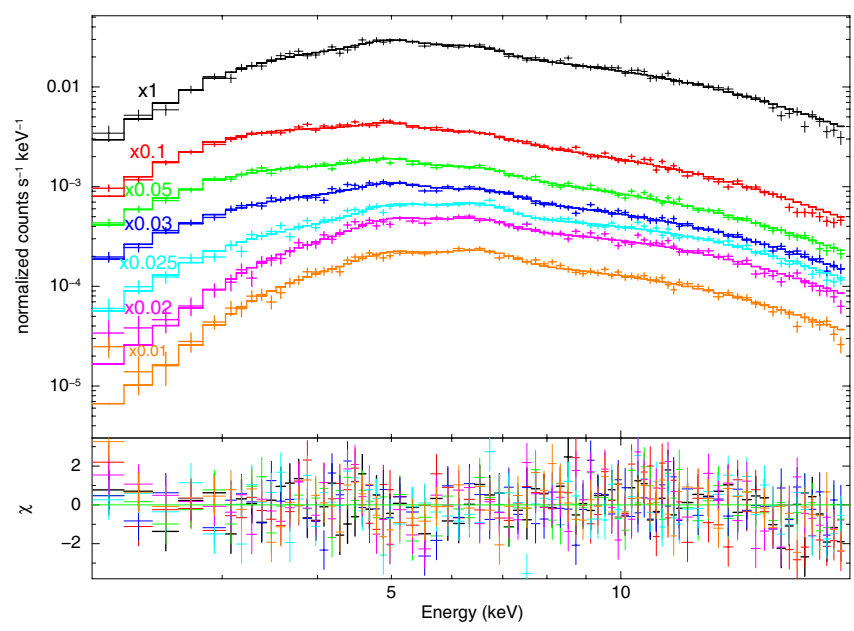

Fig. 9. Orbital phase-resolved spectra of the standard sample fit with a partial covering model, when significant (see text). Top panel (from top to bottom): data and folded model of the spectra in phase bins from $\phi_{1}$ to $\phi_{7}$. Bottom panel: residuals for the best-fit model. Symbols are the same as in Fig. 8. See Table 4 for all spectral parameters.

a good fit. For the standard sample the partial covering model is only marginally acceptable, while the cutoff power-law describes the data well.

As a result of the inhomogeneous environment in which the NS travels along the binary orbit, our phase-averaged results are not straightforward in terms of physical interpretation. Instead, these results were used to avoid unphysical best-fit solutions of the orbital phase-resolved spectra, fixing some of the model parameters to their phase-averaged value (see Sects. 6.2.2 and 6.2.3).

\subsubsection{Double-peaked sample phase-resolved spectroscopy}

Fitting the phase-resolved spectra of the double-peaked sample with a cutoff power-law results in orbital modulation of the photon index (see Table 3), which has never been observed in Vela X-1 to the best of our knowledge. The spectrum becomes harder at the inferior conjunction ( $Y$ phase bin) than at earlier and later phases ( $X$ and $Z$ phase bins, respectively). Contour plots for $N_{\mathrm{H}}$ and $\Gamma$ (shown in Fig. 6) appear clearly distinct from one phase bin to another, indicating that the modulation is not due to the intrinsic coupling between the two parameters.

Prat et al. (2008) reported photon index modulation along the binary orbit of HMXB IGR J19140+0951. These authors ascribed the observed orbital modulation to a variable visibility of the region that produces the soft excess. Since we do not observe any modulation of the blackbody component, our study does not support this interpretation. To the best of our knowledge, we did not find any reliable physical interpretation for this phenomenon.

Modeling with partial covering does not show an orbital modulation of the photon index. This model results in absorption increase along the orbital phase (see Sect. 6.2.3 for a more comprehensive discussion of this result) and in a partial covering component with high covering fraction needed at the $Y$ phase bin, which is not required at earlier or later phases, however (see Table 3). A possible interpretation of these results is given by the oscillating behavior of the accretion wake (Blondin et al. 1990). Because of the orbit-to-orbit variations of the ambient wind, the tail of the accretion wake is expected to oscillate with respect to our line of sight. Averaging this behavior over several orbital periods would result in a large covering fraction, where 
the wobbling accretion wake simulates an inhomogeneous absorbing environment.

A different interpretation might be that an intrinsically inhomogeneous structure is present around the inferior conjunction and partially covers the X-ray source. Inhomogeneous stellar winds from supergiant stars (so-called clumps) are expected (Runacres \& Owocki 2005) and observed (Goldstein et al. 2004, and references therein), and have been suggested as the cause for Vela X-1 flaring activity (see Kreykenbohm et al. 2008 and Martínez-Núñez et al. 2014). The timescale on which clumps hit the X-ray pulsar is on the order of the wind's dynamic timescale

$$
\frac{0.5 R_{\mathrm{B}}}{v_{\infty}} \sim \frac{10^{11} \mathrm{~cm}}{1100 \mathrm{~km} \mathrm{~s}^{-1}} \sim 1000 \mathrm{~s}
$$

where $0.5 R_{\mathrm{B}}$ is the average separation between clumps (Sundqvist et al. 2010). Thus, if we consider the X-ray source on longer timescales, the resulting effect is a partially covered spectrum. However, if the partial covering is due to the inhomogeneous structure of the ambient wind, no modulation of this component with orbital phase would occur. In contrast, our results underline the necessity of partial covering only for the $Y$ phase bin spectra, where the obscuration effect of the accretion wake is maximized. Hydrodynamical simulations (Blondin et al. 1990, 1991) revealed that the accretion wake is composed of compressed filaments of gas and rarefied bubbles. Moreover, recent models also showed that density perturbations in stellar winds of OB stars only develop at distances larger than the NS orbital radius in Vela X-1 $\left(r \sim 1.1 R_{\star}\right.$, see Fig. 2 in Sundqvist \& Owocki 2013). Therefore, intrinsic stellar wind clumps around the $X$-ray pulsar could be not structured enough to trigger the observed high X-ray flux variability. Instead, the inhomogeneous structure characteristic of the accretion wake trailing the NS indicates that the compact object could be able to perturb the ambient wind such that clumps are formed at its passage. The sphere of influence of the X-ray pulsar is dictated by its accretion radius, $r_{\text {acc }} \sim 10^{11} \mathrm{~cm}$ (Feldmeier et al. 1996) and by the Strömgren sphere defined by the photoionization parameter $\xi=L_{x} /\left(n R_{\text {ion }}^{2}\right)$, which leads (see Sect. 6.1 for parameter definitions and values) to a photoionization radius $R_{\text {ion }} \sim 0.2 R_{B} \sim 6 \times 10^{11} \mathrm{~cm} \sim r_{\text {acc }}$. Since the orbital velocity of the NS is $v_{\text {orbit }} \approx 280 \mathrm{~km} \mathrm{~s}^{-1}$, the perturbation timescale is about

$t_{\text {pert }}=\frac{R_{\text {ion }}}{v_{\text {orbit }}} \approx 2 \times 10^{4} \mathrm{~s} \sim 5.5 \mathrm{~h}$

which can be considered as an upper limit for the X-ray variability induced by accretion of locally formed clumps. This is consistent with observations which showed that flares in Vela X-1 last from $10^{2} \mathrm{~s}$ up to $10^{4} \mathrm{~s}$ (see Ducci et al. 2009; Martínez-Núñez et al. 2014, and references therein).

Even though the inhomogeneous structure characteristic of the accretion wake might be a possible cause for the observed partial covering around the inferior conjunction, it cannot be directly probed within the context of our MAXI analysis, which only allows inferring the long-term properties of the source averaged over several orbital phase bins.

High-resolution spectroscopic observations carried out at critical orbital phases are necessary in future to distinguish among different models and constrain stellar wind scenarios and accretion modalities in Vela X-1.

\subsubsection{Standard sample phase-resolved spectroscopy}

Modeling phase-resolved spectra of the standard sample with a cutoff power-law leads to a hardening of the spectra around the inferior conjunction of about $\Delta \Gamma \sim 0.35$. As we pointed out in Sect. 6.2.2, a physical mechanism for such an orbital dependence of the photon index is not known and is difficult to explain.

On the other hand, fitting the spectra with a partial covering model shows that the neutral matter column density $N_{\mathrm{H}}$ increases toward later phases, varying from $\sim 10^{22}$ after the egress $\left(\phi_{1}\right)$, to $\sim 10^{23} \mathrm{~cm}^{-2}$ before the ingress $\left(\phi_{7}\right)$. This agrees with earlier studies of Vela X-1 (see, e.g., Nagase 1989), and a similar phenomenon has been observed in other HMXBs as well, for instance, in 4U 1700-37 (Haberl et al. 1989). Based on the analysis of both optical (Carlberg 1978; Kaper et al. 1994) and X-ray data (Feldmeier et al. 1996), it has been shown that the accretion wake is not adequate to explain the later phase $(\phi>0.5)$ absorption, and that a more extended photoionization wake is necessary.

When this model is applied, the spectral photon index modulation along the orbital phase is only marginal. Based on the best-fit results, we can adopt here a similar interpretation as for the double-peaked sample results, that is, an inhomogeneous ambient medium that produces strong absorption of the X-ray emission and whose absorption efficiency depends on the orbital phase (see Sect. 6.2.2). The column density shows a local peak of $N_{\mathrm{H}}^{\mathrm{pc}} \sim 3 \times 10^{23} \mathrm{~cm}^{-2}$ around the inferior conjunction, similar to what is observed for the double-peaked sample, strengthening the connection with the accretion wake. Moreover, this sample needs the partial covering component also to fit the spectrum of the phase bin $\phi_{2}$, which is located around the NS greatest eastern elongation (see Fig. 1). Regardless of whether the partial covering is due to a wobbling or an inhomogeneous accretion wake, these results indicate at a wake that affects the observations at earlier phases than the inferior conjunction.

We therefore conclude that the absorbing (and likely inhomogeneous) material that partially covers the X-ray pulsar might be due to a different influence of the accretion wake at different orbital phases.

\section{Summary}

We have presented the results of a very long MAXI observation of Vela X-1, which allowed us to perform detailed spectral (phase-averaged and phase-resolved, in 2-20 keV) and light curve (in 4-20 keV) analysis. Our results can be summarized as follows:

- About $15 \%$ of the orbital light curves in Vela X-1 shows a double-peaked orbital profile, with a $\sim 1.5$ day dip around the inferior conjunction in the 4-10 keV and 10-20 keV energy bands.

- Explaining the dip around the inferior conjunction by absorption alone requires column density values of about $2 \times$ $10^{24} \mathrm{~cm}^{-2}$, which are not observed in our analysis.

- The dip in the double-peaked sample might be produced by considering contribution from Thomson scattering by an ionized accretion wake that is elongated toward the observer during the transit. Intrinsic variability of the stellar wind leads to strong variations of the accretion wake, including its ionization degree, thus to alternating double-peaked and standard profiles.

- When fit with a cutoff power-law model, the double-peaked and the standard samples both show spectral hardening 
around the inferior conjunction (by $\sim 0.3$ in terms of the photon index). We did not find any reliable physical interpretation for this phenomenon, which likely indicates that the model is inadequate for Vela X-1.

- Orbital photon index modulation is avoided if a partial covering component is used around the inferior conjunction. This component indicates a highly inhomogeneous environment and local column density peak values of $\sim 3 \times 10^{23} \mathrm{~cm}^{-2}$ around the inferior conjunction (orbital phases around the eclipse were not analyzed).

- Our results suggest either a wobbling or an inhomogeneous accretion wake as the source of the partial covering around the inferior conjunction. Gravitational and radiative effects from the X-ray pulsar may be responsible of density inhomogeneities in the ambient wind that successively feed the observed high X-ray variability. Moreover, the absorption properties of the accretion wake seem to take place at earlier phases than the inferior conjunction.

- Some of the analyzed orbital phase-bin spectra do not need a partial covering component. Therefore, any spectral analysis of Vela X-1 needs to consider the orbital phase at which the observation has been carried out.

Acknowledgements. We gratefully acknowledge the anonymous referee for numerous comments that greatly improved the manuscript. This work is supported by the International Program Associate (IPA) of RIKEN, Japan, and by the Bundesministerium für Wirtschaft und Technologie through the Deutsches Zentrum für Luft- und Raumfahrt e.V. (DLR) under the grant number FKZ 50 OR 1204. C.M. gratefully thanks the entire MAXI team for the collaboration and hospitality in RIKEN. C.M. also thanks V. Doroshenko and L. Ducci (IAAT, Tübingen) for useful discussions.

\section{References}

Anders, E., \& Grevesse, N. 1989, Geochim. Cosmochim. Acta, 53, 197 Blondin, J. M., Kallman, T. R., Fryxell, B. A., \& Taam, R. E. 1990, ApJ, 356, 591

Blondin, J. M., Stevens, I. R., \& Kallman, T. R. 1991, ApJ, 371, 684

Carlberg, R. G. 1978, Ph.D. Thesis, The University of British Columbia, Canada
Chodil, G., Mark, H., Rodrigues, R., Seward, F. D., \& Swift, C. D. 1967, ApJ, 150,57

Doroshenko, V., Santangelo, A., Nakahira, S., et al. 2013, A\&A, 554, A37

Ducci, L., Sidoli, L., Mereghetti, S., Paizis, A., \& Romano, P. 2009, MNRAS, 398, 2152

Eadie, G., Peacock, A., Pounds, K. A., et al. 1975, MNRAS, 172, 35P

Feldmeier, A., Anzer, U., Boerner, G., \& Nagase, F. 1996, A\&A, 311, 793

Fürst, F., Kreykenbohm, I., Pottschmidt, K., et al. 2010, A\&A, 519, A37

Fürst, F., Pottschmidt, K., Wilms, J., et al. 2014, ApJ, 780, 133

Goldstein, G., Huenemoerder, D. P., \& Blank, D. 2004, AJ, 127, 2310

Haberl, F., White, N. E., \& Kallman, T. R. 1989, ApJ, 343, 409

Hickox, R. C., Narayan, R., \& Kallman, T. R. 2004, ApJ, 614, 881

Jackson, J. C. 1975, MNRAS, 172, 483

Kaper, L., Hammerschlag-Hensberge, G., \& van Loon, J. T. 1993, A\&A, 279, 485

Kaper, L., Hammerschlag-Hensberge, G., \& Zuiderwijk, E. J. 1994, A\&A, 289, 846

Kreykenbohm, I., Wilms, J., Kretschmar, P., et al. 2008, A\&A, 492, 511

Manousakis, A., Walter, R., \& Blondin, J. M. 2012, A\&A, 547, A20

Martínez-Núñez, S., Torrejón, J. M., Kühnel, M., et al. 2014, A\&A, 563, A70

Matsuoka, M., Kawasaki, K., Ueno, S., et al. 2009, PASJ, 61, 999

Mauche, C. W., Liedahl, D. A., Akiyama, S., \& Plewa, T. 2008, in AIP Conf. Ser. 1054, ed. M. Axelsson, 3

Mihara, T., Nakajima, M., Sugizaki, M., et al. 2011, PASJ, 63, 623

Morrison, R., \& McCammon, D. 1983, ApJ, 270, 119

Nagase, F. 1989, PASJ, 41, 1

Naik, S., Mukherjee, U., Paul, B., \& Choi, C. S. 2009, Adv. Space Res., 43, 900

Odaka, H., Khangulyan, D., Tanaka, Y. T., et al. 2013, ApJ, 767, 70

Prat, L., Rodriguez, J., Hannikainen, D. C., \& Shaw, S. E. 2008, MNRAS, 389, 301

Prinja, R. K., Barlow, M. J., \& Howarth, I. D. 1990, ApJ, 361, 607

Rawls, M. L., Orosz, J. A., McClintock, J. E., et al. 2011, ApJ, 730, 25

Runacres, M. C., \& Owocki, S. P. 2005, A\&A, 429, 323

Rybicki, G. B., \& Lightman, A. P. 1979, Radiative processes in astrophysics (New York: Wiley-Interscience)

Schanne, S., Götz, D., Gérard, L., et al. 2007, in ESA SP, 622, 479

Sidoli, L., Paizis, A., Fürst, F., et al. 2015, MNRAS, 447, 1299

Smith, M. A. 2001, ApJ, 562, 998

Sugizaki, M., Mihara, T., Serino, M., et al. 2011, PASJ, 63, 635

Sundqvist, J. O., \& Owocki, S. P. 2013, MNRAS, 428, 1837

Sundqvist, J. O., Puls, J., \& Feldmeier, A. 2010, A\&A, 510, A11

Tomida, H., Tsunemi, H., Kimura, M., et al. 2011, PASJ, 63, 397

van Kerkwijk, M. H., van Paradijs, J., Zuiderwijk, E. J., et al. 1995, A\&A, 303, 483

Watanabe, S., Sako, M., Ishida, M., et al. 2006, ApJ, 651, 421

Watson, M. G., \& Griffiths, R. E. 1977, MNRAS, 178, 513 